\title{
Chitosan-MgO Nanocomposite: One Pot Preparation and Its Utility as an Ecofriendly Biocatalyst in the Synthesis of Thiazoles and $[1,3,4]$ thiadiazoles
}

\author{
Sayed M. Riyadh ${ }^{1,2}$, Khaled D. Khalil ${ }^{2,3, *(D)}$ and Ateyatallah Aljuhani ${ }^{1}$ \\ 1 Department of Chemistry, Faculty of Science, Taibah University, Al-Madinah Al-Mounawrah 30002, \\ Saudi Arabia; riyadh1993@hotmail.com (S.M.R.); ateyatallah@hotmail.com (A.A.) \\ 2 Department of Chemistry, Faculty of Science, Cairo University, Giza 12613, Egypt \\ 3 Department of Chemistry, Faculty of Science, Taibah University, Yanbu 46423, Saudi Arabia \\ * Correspondence: khd.khalil@yahoo.com
}

Received: 17 October 2018; Accepted: 4 November 2018; Published: 8 November 2018

check for updates

\begin{abstract}
A chitosan-MgO hybrid nanocomposite was prepared using a simple chemical precipitation method and characterized using Fourier transform spectroscopy (FTIR), elemental analysis (EDX), and scanning electron microscopy (SEM). The nanocomposite was served as a powerful ecofriendly basic catalyst under microwave irradiation in the synthesis of two novel series of 5-arylazo-2-hydrazonothiazoles $\mathbf{4 a - j}$ and 2-hydrazono[1,3,4]thiadiazoles $\mathbf{8 a -} \mathbf{d}$, incorporating a sulfonamide group. The structures of the synthesized products were elucidated by spectral data and elemental analyses. Also, their yield percentages were calculated using triethylamine (as a traditional catalyst) and chitosan- $\mathrm{MgO}$ nanocomposite (as a green recyclable catalyst) in a comparative study.
\end{abstract}

Keywords: chitosan-MgO nanocomposite; heterogeneous catalysis; ethylidenethiosemicarbazides; thiazoles; thiadiazoles

\section{Introduction}

In the past decade, green chemistry has been developed from a variety of research ideas such as atom economy and heterogeneous catalysis in the last few decades leading up to the 1990s, and in several successful attempts and efforts to overcome the problems of chemical pollution and resource depletion. On the other hand, nanosized materials are of great importance due to their large exposed surface area, high absorbability, and high catalytic efficiency. Nanosized magnesium oxides were being multitalented basic catalysts for many organic reactions. Recently, $\mathrm{MgO}$ nanoparticles can be used for the synthesis of pyranopyrazoles [1], aminochromenes [2], and dihydropyridines [3] via multicomponent reactions. The main drawbacks to the potential use of $\mathrm{MgO}$ nanoparticles as a basic catalyst are their difficult separation and reusability since the utilized catalyst could not recover quantitatively and the pure products should be obtained after extensive purification processes. Chitosan (CS) is a natural polysaccharide that is commercially produced via the alkaline hydrolysis of chitin. Although this biopolymer has many advantages, such as its renewability, biocompatibility, and biodegradability, its utility is limited in its unmodified form. For a long time, chitosan was used as an ecofriendly basic catalyst in some organic reactions [4-7]. However, the major problem of its use is the limited basic properties (weak catalytic activity) and its high swelling properties and gel formation renders its separation and recovery very difficult. To overcome these drawbacks, a chitosan-MgO nanocomposite [8] could be used as a novel basic heterogeneous catalyst in the form of a solid film. A chitosan-MgO nanocomposite is a three-dimensional, cross-linked, polymeric matrix of chitosan (with active $\mathrm{NH}$ and $\mathrm{OH}$ functional groups) incorporating magnesium oxide nanoparticles (Figure 1) [9]. 

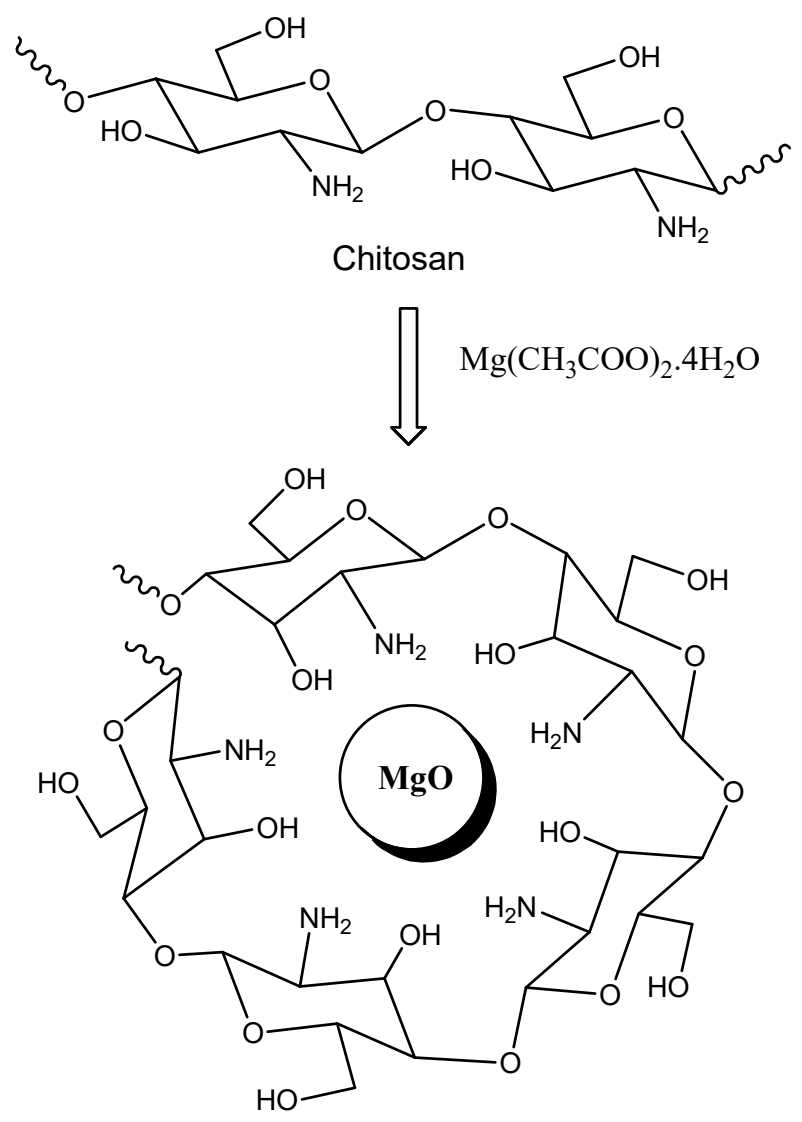

Figure 1. Structure of a chitosan-MgO nanocomposite.

The biocatalyst is stable enough such that it can be recovered and used more than four times without a loss in its catalytic activity. As a result of these studies, the chitosan-MgO biocatalyst was found to be ecofriendly, biocompatible, an efficient green catalyst, and a high economic impact material that will find a lot of potential catalytic applications, especially in the base catalyzed organic transformations. This heterogeneous hybrid nanocomposite is used in the form of films to increase its catalytic activity by increasing its surface/volume ratio, and consequently it can be easily recycled and reused several times with the same catalytic efficiency [10].

Moreover, thiazoles display a diversity of therapeutic activities such as being a non-steroidal anti-inflammatory drug (meloxicam), antiretroviral drug (ritonavir), antiprotozoal agent (nitazoxanide) [11], and antioxidant agent [12]. Also, [1,3,4]thiadiazoles with a sulfonamide group were incorporated in many marketing drugs including acetazolamide and methazolamide [13]. In this study, we are reporting an efficient green protocol and simple synthetic routes of a new series of hydrazonothiazoles and hydrazono $[1,3,4]$ thiadiazoles in the presence of chitosan-MgO nanocomposite as a basic ecofriendly biocatalyst under microwave irradiations. Moreover, the efficiency of this biocatalyst and its reusability was examined.

\section{Materials and Methods}

Melting points were measured on an electrothermal Gallenkamp capillary apparatus (Leicester, UK) and are uncorrected. Elemental analyses were carried out at the Microanalytical Center of Cairo University, Giza, Egypt. The IR spectra were recorded on a Pye-Unicam SP300 Instrument (Cambridge, UK) in potassium bromide discs. The ${ }^{1} \mathrm{H}$ NMR (nuclear magnetic resonance) and ${ }^{13} \mathrm{C}$ NMR of the newly synthesized compounds in DMSO- $d_{6}$ were measured on a Varian Mercury VXR-300 spectrometer (Varian, Karlsruhe, Germany) at $300 \mathrm{MHz}$ for ${ }^{1} \mathrm{H}$ NMR and $75 \mathrm{MHz}$ for ${ }^{13} \mathrm{C}$ NMR) and the chemical shifts were related to that of the solvent. The mass spectra were recorded 
on a GCMSQ1000-EX Shimadzu (Tokyo, Japan) and GCMS 5988-A HP spectrometers (Shimadzu, Tokyo, Japan) where the ionizing voltage was $70 \mathrm{eV}$. Microwave experiments were carried out using CEM Discover Labmate microwave apparatus (Discover, SP, NC, USA, $300 \mathrm{~W}$ ). The starting materials 2-\{1-[4-((4-methylphenyl)sulfonamide) phenyl]ethylidine\}thiosemicarbazide 1 [14], $\alpha$-keto hydrazonoyl halides $\mathbf{2 a - j}[\mathbf{j}-18]$, and arenecarbohydrazonoyl halides $\mathbf{5 a}-\mathbf{d}[19,20]$ were prepared as reported in literature.

\subsection{Preparation of Heterogeneous Catalyst (Chitosan-MgO Hybrid Nanocomposite)}

The hybrid CS-MgO nanocomposite was prepared using a one pot solution casting method. In an experiment, $2 \mathrm{~g}$ of chitosan powder (medium molecular weight; 85\% DDA) was dissolved in $100 \mathrm{~mL}$ of $2 \%(v / v)$ aqueous acetic acid solution under stirring for $12 \mathrm{~h}$ at room temperature. To this solution, $2 \mathrm{~g}$ of magnesium acetate tetrahydrate, $\mathrm{Mg}\left(\mathrm{CH}_{3} \mathrm{COO}\right)_{2} \cdot 4 \mathrm{H}_{2} \mathrm{O}$ (M0631 Sigma-Aldrich, St. Louis, $\mathrm{MO}$, USA) were added and the mixture was again re-stirred for $12 \mathrm{~h}$ till a clear solution was obtained. The resulting viscous solution was cast in a Teflon Petri dish and dried overnight at room temperature. The Petri dish was then immersed into a $0.2 \mathrm{M}$ sodium hydroxide solution for neutralization and phase separation. The solid films were dried in an oven adjusted at $80^{\circ} \mathrm{C}$ for $6 \mathrm{~h}$. Finally, the produced solid films were purified by washing with methanol several times and again were dried in an oven at $60^{\circ} \mathrm{C}$ for $2 \mathrm{~h}$. Furthermore, a pure chitosan film, without $\mathrm{MgO}$, was prepared in a similar way for comparative study.

2.2. Reactions of 2-\{1-[4-((4-Methylphenyl)sulfonamide)phenyl]ethylidine\}thiosemicarbazide (1) with $\alpha$-Keto Hydrazonoyl Chlorides $\mathbf{2 a - j}$ or N-Aryl Arenecarbohydrazonoyl Halides 5a-d

\subsubsection{Method A}

A mixture of 2-\{1-[4-((4-methylphenyl)sulfonamide)phenyl]ethylidine\}thiosemicarbazide (1) $(0.362 \mathrm{~g}, 1 \mathrm{mmol})$ in dry dioxane $(15 \mathrm{~mL})$, containing $0.1 \mathrm{~g}$ of triethylamine, and $\alpha$-keto hydrazonoyl chlorides $\mathbf{2 a - j}$ or $N$-aryl arenecarbohydrazonoyl halides $5 \mathbf{a}-\mathbf{d}$ ( 1 mmol of each) was irradiated using microwave irradiation (MW) at $300 \mathrm{~W}$ in a closed Teflon vessel until all the starting material was consumed (30-40 $\mathrm{min}$ as monitored by thin layer chromatography, TLC). The solvent was evaporated and the residue was poured into an ice $/ \mathrm{HCl}$ mixture. The precipitate was filtered, washed with water, and crystallized from methanol to give products $4 \mathbf{a}-\mathbf{j}$ or $\mathbf{8} \mathbf{a}-\mathbf{d}$.

\subsubsection{Method B}

The procedure was similar to Method A, using a chitosan-MgO nanocomposite $(0.1 \mathrm{~g})$ instead of trimethylamine. After completion of the reaction, the hot solution was filtered to remove chitosan-MgO nanocomposite and the filtrate was poured into an ice $/ \mathrm{HCl}$ mixture, and the precipitate was filtered, washed with water, and crystallized from methanol to give products $\mathbf{4 a - j}$ or $\mathbf{8 a}-\mathbf{d}$ (see Supplementary Materials for analyses of the prepared compounds).

\section{Results and Discussion}

\subsection{Preparation and Characterization of Chitosan-MgO Nanocomposite Films}

The chitosan-MgO nanocatalyst was prepared via incorporation of the $\mathrm{MgO}$ nanoparticles in the chitosan matrix through a modified one pot solution casting method. The chitosan solution in acetic acid was treated with magnesium acetate tetrahydrate, then was subjected to evaporation of the solvent at room temperature. The resulting solid film was soaked in sodium hydroxide solution to achieve the phase separation. 


\subsubsection{FTIR Spectra}

FTIR (Fourier-Transform infrared) spectra of chitosan and chitosan-MgO nanocomposite showed that the main functional groups of chitosan clearly appeared at $v=3370 \mathrm{~cm}^{-1}$ (broad band of hydrogen bonded $\mathrm{OH}$ - groups), $2875 \mathrm{~cm}^{-1}$ (C-H bond; $\mathrm{CH}_{3}$ groups), $1660 \mathrm{~cm}^{-1}$ (amide carbonyl groups), $1379 \mathrm{~cm}^{-1}$ (bending vibration of $\mathrm{CH}_{2}$ groups), and $1060 \mathrm{~cm}^{-1}$ (asym. vibration of $\mathrm{C}=\mathrm{O}$ ) (Figure 2). These bands are considered as evidence for the maintenance of the chitosan structure features even after the incorporation of the $\mathrm{MgO}$ nanoparticles inside the polymer matrix. Also, the absence of acetic acid bands in the spectrum indicated that the films were washed enough and neutralized completely upon sodium hydroxide treatment (see Section 2.1). Moreover, only small acceptable shift in the bands of chitosan-MgO nanocomposite was attributed to the influence of the incorporation of $\mathrm{MgO}$ nanoparticles. This shift in bands was familiar as result of chitosan with metal oxides [21]. The latter shifts are shown, especially at bands of $\mathrm{NH}$ and $\mathrm{OH}$ groups, which is evidence for the $\mathrm{H}$-bonding interaction of these group with $\mathrm{MgO}$ molecules.

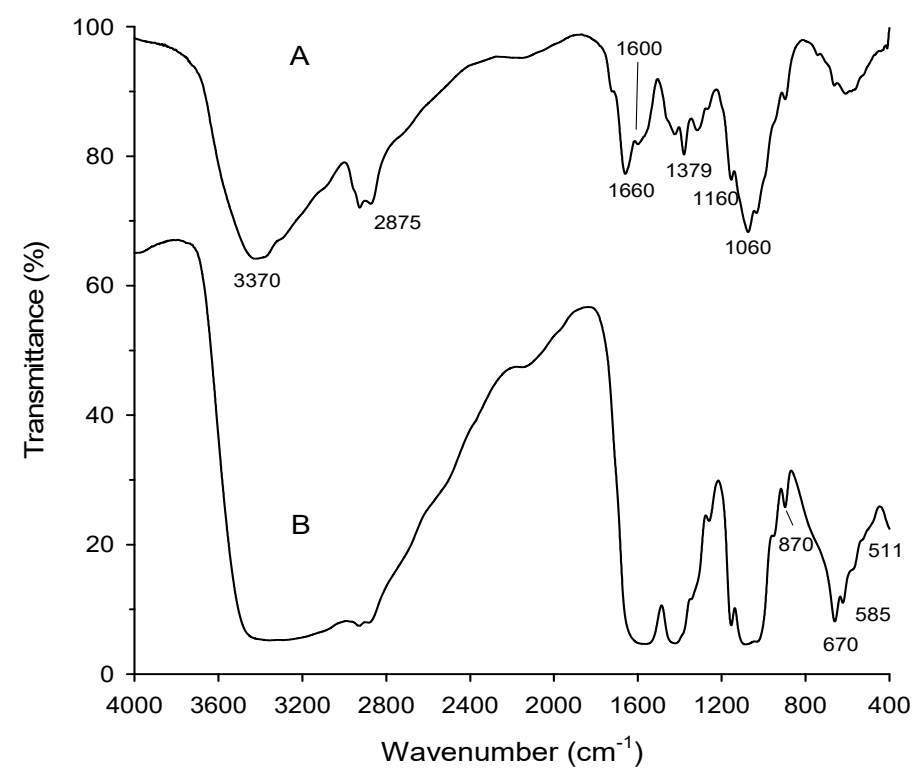

Figure 2. FTIR of chitosan (A) and the chitosan-MgO nanocomposite (B).

\subsubsection{FESEM Analysis}

FESEM (Field Emission Scanning Electron Microscope) was used to analyze the morphology and size distribution of the $\mathrm{MgO}$ nanoparticles that were incorporated in the polymer matrix. FESEM micrographs of the pure chitosan (A) and that of the hybrid films with magnesia particles $10 \mathrm{wt} \%$ (B) are shown in (Figure 3). The obtained surface of the pure chitosan matrix was found to be homogenous and looks smoothly to a great extent. The $\mathrm{MgO}$ nanoparticles developed as white spots that distributed homogeneously over the surface of the polymer matrix. On the other hand, the muddled surface was due to adsorption of the polymer layers on the particles surface. The average size of the $\mathrm{MgO}$ particles was found to be approximately $6-11 \mathrm{~nm}$ for $10 \mathrm{wt} \%$ as the particles size slightly decreased with increasing magnesia content. Moreover, EDX measurements for the solid chitosan-MgO nanocomposite confirmed the presence of magnesia within the hybrid film, as shown in (Figure 4). 


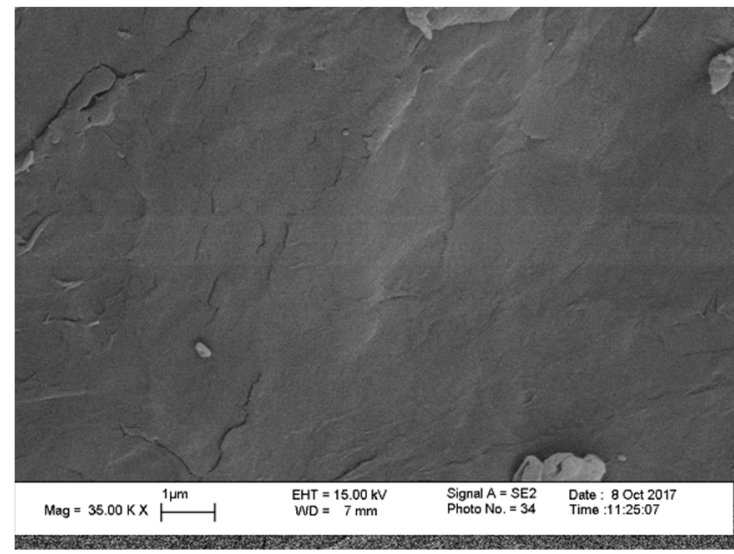

(A)

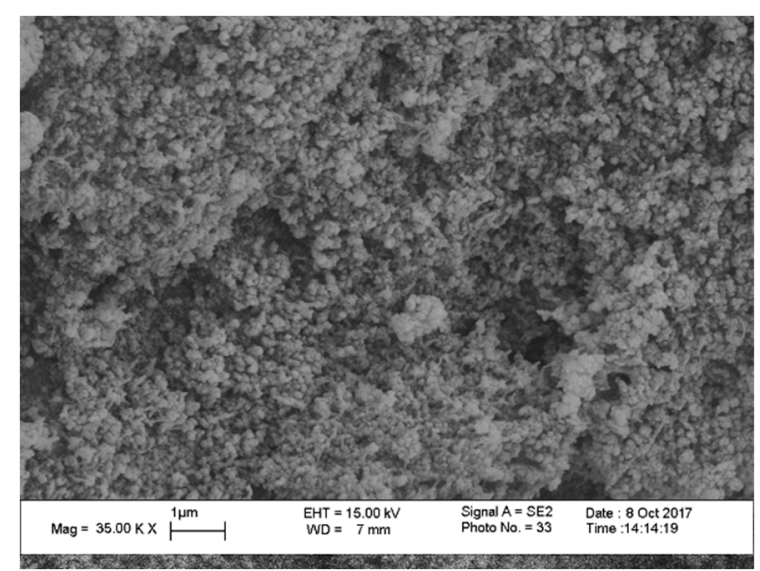

(B)

Figure 3. FESEM images of chitosan (A), and chitosan-MgO $10 \mathrm{wt} \%$ (B).

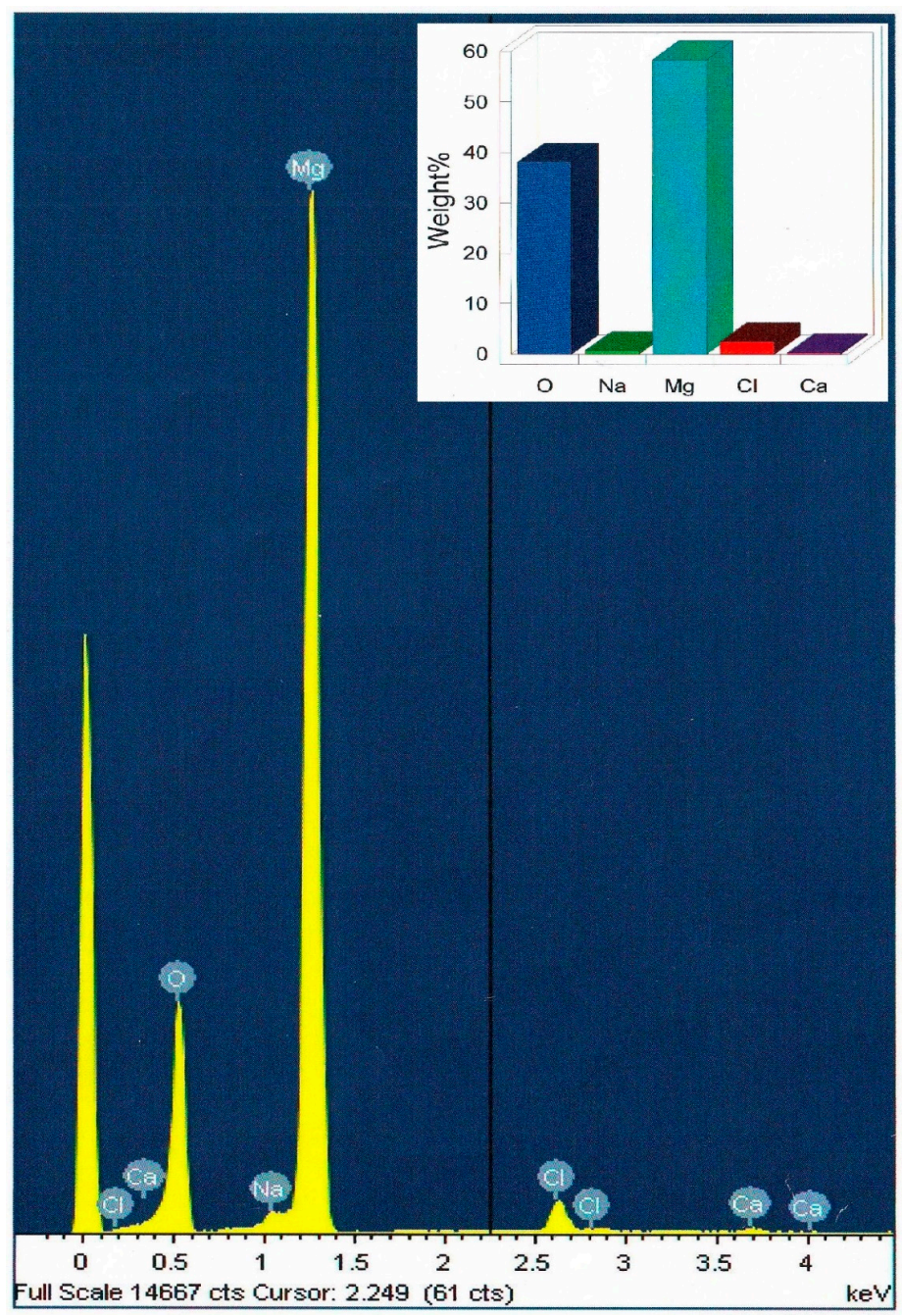

Figure 4. EDX spectra of the $10 \mathrm{wt} \%$ chitosan-MgO nanocomposite hybrid film. Inset is the quantitative result.

\subsection{Optimal Catalyst Loading}

In order to estimate the proper catalyst loading, a model irradiation reaction of ethylidinethiosemicarbazide 1 with $\alpha$-keto hydrazonoyl chlorides $2 \mathbf{a}$ in dioxane, and in the presence of $1,5,10,15$, and $20 \mathrm{wt} \%$ of chitosan-MgO nanocomposites as basic catalysts, under the same conditions 
was conducted affording the respective hydrazonothiazoles $4 \mathbf{a}$ (Figure 5). The results showed that the optimal catalyst loading was $10 \mathrm{wt} \%$ Moreover, the catalyst was reused four time and the results also showed that the biocatalyst could be reused as such without significant loss in its catalytic activity (Table 1).

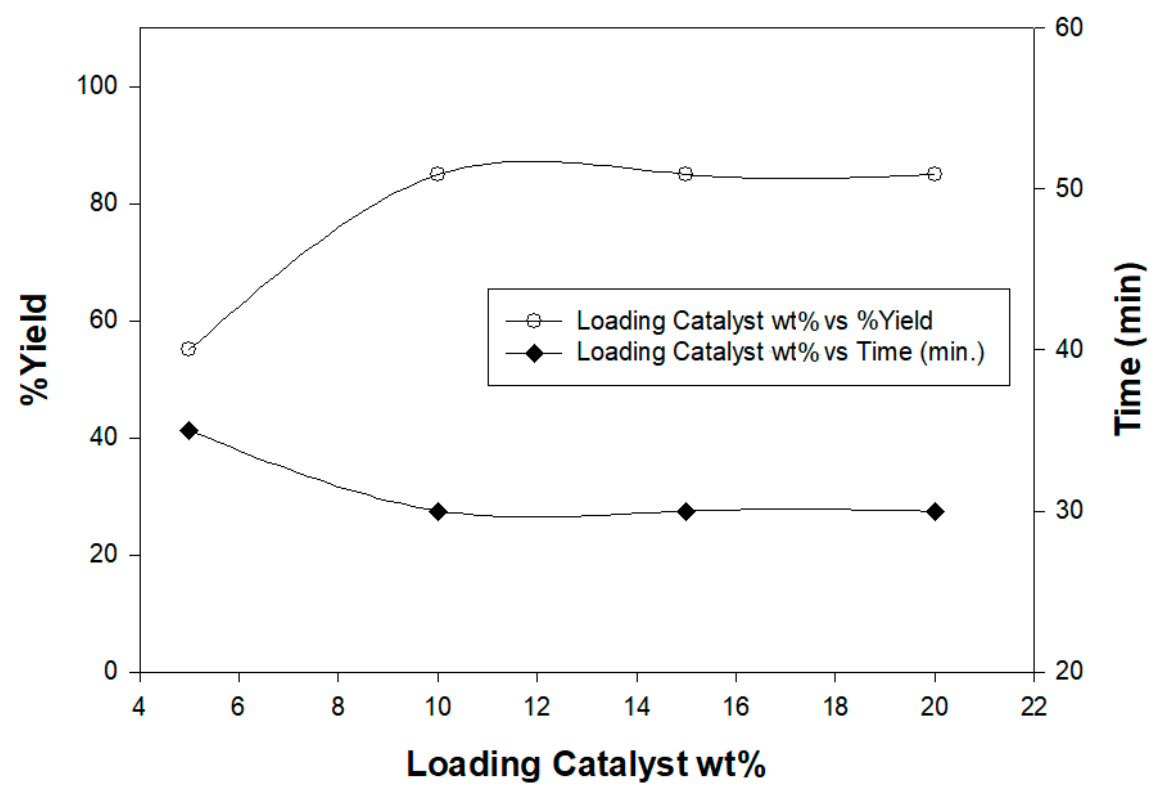

Figure 5. Optimization of the chitosan graft copolymer as a basic catalyst.

Table 1. Recyclability of the chitosan graft copolymer as basic catalyst.

\begin{tabular}{cccccc}
\hline State of Catalyst & Fresh Catalyst & Recycled (1) & Recycled (2) & Recycled (3) & Recycled (4) \\
\hline Product 4a (\%Yield) & 85 & 84 & 83 & 83 & 82 \\
\hline
\end{tabular}

\subsection{Synthesis of Thiazoles and [1,3,4]thiadiazoles Using Cs-MgO Nanocomposite}

Irradiation of thiosemicarbazone $\mathbf{1}$ with 2-oxo- $N$-arylpropanehydrazonoyl chlorides $\mathbf{2 a} \mathbf{a}-\mathbf{j}$ in dioxane, in the presence of triethylamine or the chitosan-MgO nanocomposite as a basic catalyst, furnished 4-methyl-5-arylazo-2-hydrazonothiazoles $4 \mathbf{a}-\mathbf{j}$ (Scheme 1). At the outset, identification of the best basic catalyst (triethylamine or chitosan- $\mathrm{MgO}$ nanocomposite) was examined under microwave irradiations (Table 2).

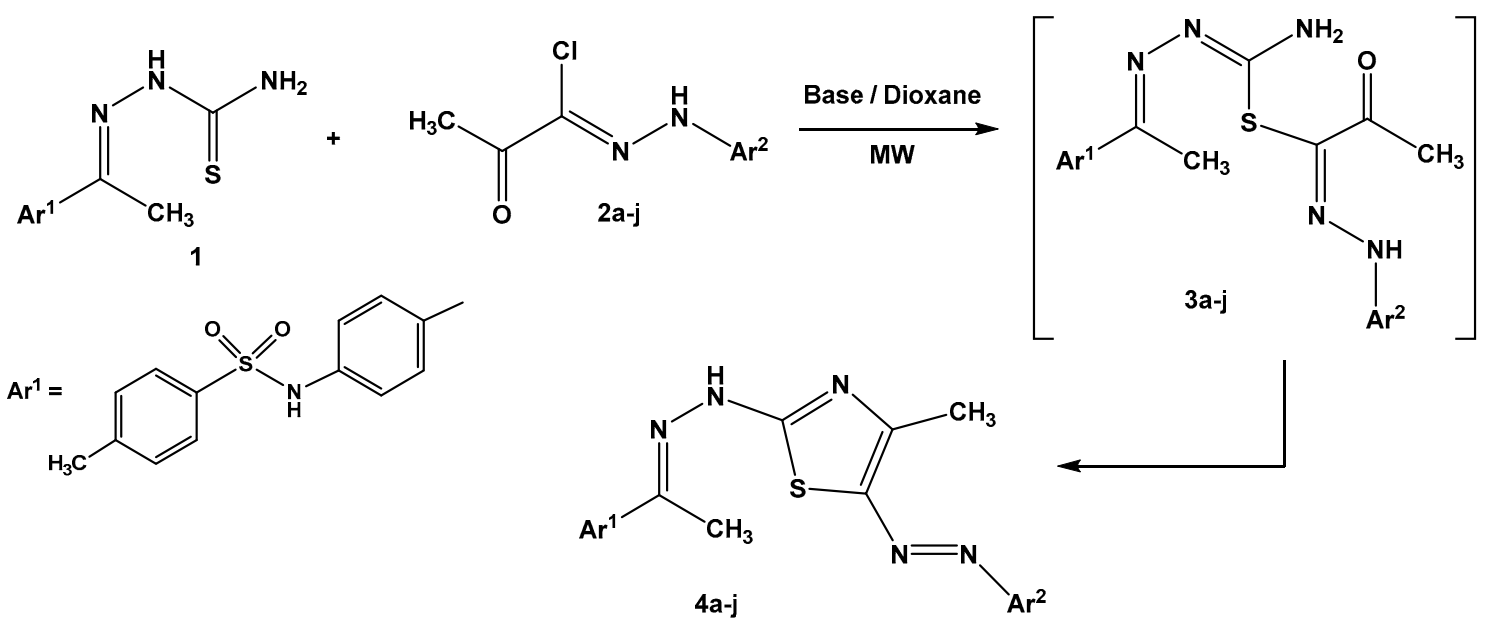

Scheme 1

Scheme 1. Synthesis of hydrazonothiazoles $\mathbf{4 a - j}$. 
Table 2. Effect of the nature of basic catalyst on the yield of the products $4 \mathbf{a}-\mathbf{j}$.

\begin{tabular}{ccccc}
\hline \multirow{2}{*}{ Compound Number } & $\mathbf{A r}^{2}$ & Time (min) & \multicolumn{2}{c}{ Yield (\%) } \\
\cline { 3 - 5 } & & & $\mathbf{E t}_{3} \mathbf{N}$ & Chitosan-MgO Nanocomposite \\
\hline $\mathbf{4 a}$ & $\mathrm{C}_{6} \mathrm{H}_{5}$ & 30 & 76 & 85 \\
$\mathbf{4} \mathbf{b}$ & $2-\mathrm{CH}_{3} \mathrm{C}_{6} \mathrm{H}_{4}$ & 30 & 73 & 85 \\
$\mathbf{4} \mathbf{c}$ & $2-\mathrm{ClC}_{6} \mathrm{H}_{4}$ & 35 & 77 & 86 \\
$\mathbf{4}$ & $3-\mathrm{CH}_{3} \mathrm{C}_{6} \mathrm{H}_{4}$ & 30 & 82 & 96 \\
$\mathbf{4}$ & $4-\mathrm{CH}_{3} \mathrm{C}_{6} \mathrm{H}_{4}$ & 30 & 78 & 87 \\
$\mathbf{4} \mathbf{f}$ & $4-\mathrm{CH}_{3} \mathrm{OC}_{6} \mathrm{H}_{4}$ & 40 & 73 & 83 \\
$\mathbf{4 g}$ & $4-\mathrm{NO}_{2} \mathrm{C}_{6} \mathrm{H}_{4}$ & 40 & 69 & 81 \\
$\mathbf{4 h}$ & $4-\mathrm{BrC}_{6} \mathrm{H}_{4}$ & 40 & 70 & 80 \\
$\mathbf{4 i}$ & $4-\mathrm{FC}_{6} \mathrm{H}_{4}$ & 40 & 71 & 84 \\
$\mathbf{4} \mathbf{j}$ & $4-\mathrm{CH}_{3} \mathrm{COC}_{6} \mathrm{H}_{4}$ & 40 & 72 & \\
\hline
\end{tabular}

As shown in Table 2, the reaction proceeded smoothly with different substituents on the aromatic benzene ring of hydrazonoyl chlorides $\mathbf{2 a}-\mathbf{j}$. Also, the chitosan-MgO nanocomposite was a more efficient basic catalyst than triethylamine under microwave irradiation.

Elucidation of 2-hydrazonothiazoles $4 \mathbf{a}-\mathbf{j}$ structures was based on spectral data and elemental analyses. In the IR spectra, two absorption bands in the range of $v=3212-3268 \mathrm{~cm}^{-1}$ and $1578-1600 \mathrm{~cm}^{-1}$ were revealed owing to the presence of $(\mathrm{N}-\mathrm{H})$ and $(\mathrm{C}=\mathrm{N})$ groups, respectively. Also, the sulfonamide group $\left(\mathrm{SO}_{2} \mathrm{NH}\right)$ showed asymmetric and symmetric stretching signals at $v=1336-1379 \mathrm{~cm}^{-1}$ and 1136-1161 $\mathrm{cm}^{-1}$, respectively [22]. In ${ }^{1} \mathrm{H}$ NMR spectra two methyl groups bordering to hydrazone moiety $\left(\mathrm{CH}_{3}-\mathrm{C}=\mathrm{N}-\mathrm{NH}\right)$ [23] and a thiazole ring [24] were observed as singlet signals at $\delta=2.35-2.43$ and 2.43-2.59 ppm, respectively, while the NH proton of the sulfonamide group [22] was resonated at $\delta=10.43-10.92$ ppm.

Establishing the experimental feasibility of the reaction of 1 with $\alpha$-keto hydrazonoyl chlorides $\mathbf{2 a}-\mathbf{j}$ directed our attention to use $N$-aryl arenecarbohydrazonoyl halides $\mathbf{5 a - d}$, bereft of the carbonyl group. Thus, treatment of ethylidinethiosemicarbazide 1 with $N$-aryl arenecarbohydrazonoyl halides $\mathbf{5 a}-\mathbf{d}$ under the same employed conditions proceeded smoothly to give 2-hydrazono[1,3,4] thiadiazoles $\mathbf{8 a}-\mathbf{d}$ as the isolated products (Scheme 2). Also, the effect of the nature of the basic catalyst, such as triethylamine or the chitosan-MgO nanocomposite, on the percent yields of the isolated products $8 \mathbf{a}-\mathbf{d}$ was investigated (Table 3).

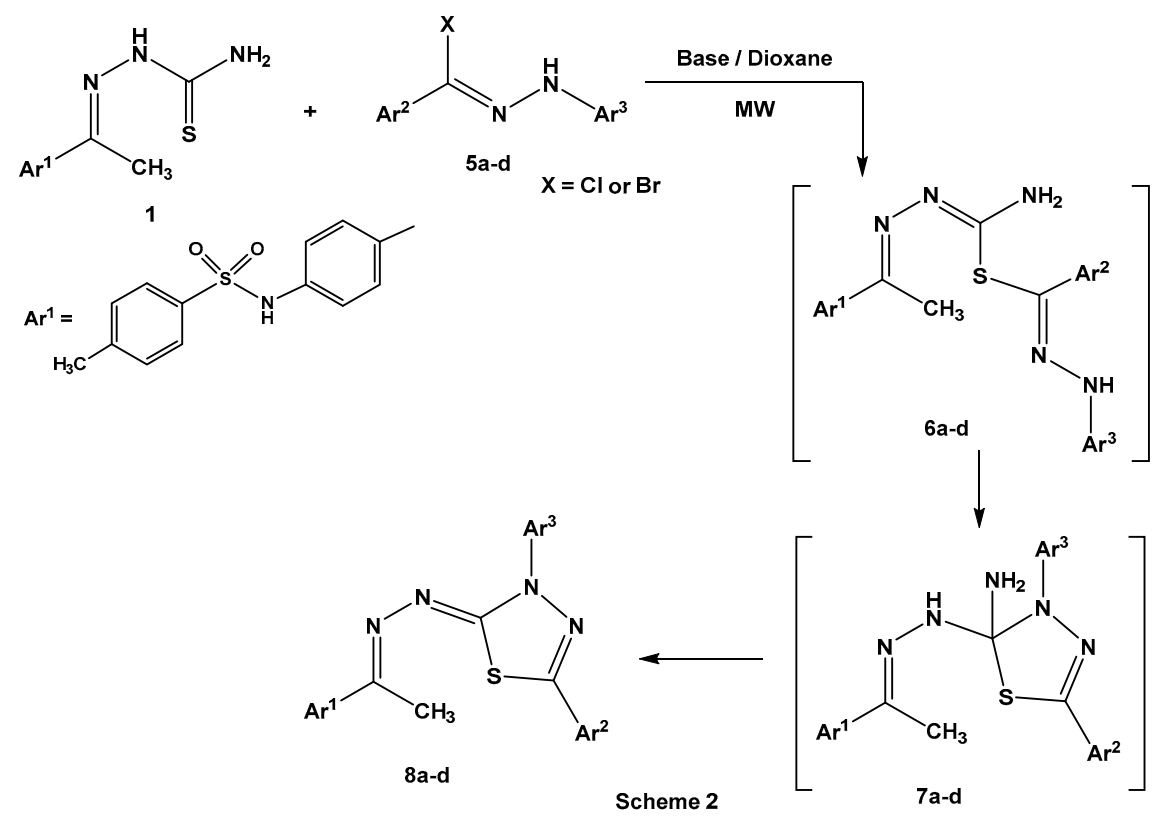

Scheme 2. Synthesis of hydrazono[1,3,4]thiadiazoles 8a-d. 
Table 3. Effect of the nature of basic catalyst on the yield of the products $8 \mathbf{a}-\mathbf{d}$.

\begin{tabular}{cccccc}
\hline \multirow{2}{*}{$\begin{array}{c}\text { Compound } \\
\text { Number }\end{array}$} & \multirow{2}{*}{$\mathbf{A r}^{2}$} & $\mathbf{A r}^{3}$ & Time (min) & \multicolumn{2}{c}{ Yield (\%) } \\
\cline { 5 - 6 } & & $\mathrm{Et}_{3} \mathbf{N}$ & Chitosan/MgO Nanocomposite \\
\hline $\mathbf{8 a}$ & $\mathrm{C}_{6} \mathrm{H}_{5}$ & $\mathrm{C}_{6} \mathrm{H}_{5}$ & 30 & 74 & 86 \\
$\mathbf{8 b}$ & $\mathrm{C}_{6} \mathrm{H}_{5}$ & $4-\mathrm{NO}_{2} \mathrm{C}_{6} \mathrm{H}_{4}$ & 35 & 76 & 88 \\
$\mathbf{8}$ & $4-\mathrm{CH}_{3} \mathrm{C}_{6} \mathrm{H}_{4}$ & $\mathrm{C}_{6} \mathrm{H}_{5}$ & 40 & 79 & 85 \\
$\mathbf{8 d}$ & $4-\mathrm{CH}_{3} \mathrm{OC}_{6} \mathrm{H}_{4}$ & $\mathrm{C}_{6} \mathrm{H}_{5}$ & 40 & 78 & 87 \\
\hline
\end{tabular}

As shown in Table 3, the percentage yields of the products $\mathbf{8 a}-\mathbf{d}$, the chitosan-MgO nanocomposite as a basic catalyst prevailed over triethylamine under microwave irradiation. The characterization of 2-hydrazono[1,3,4] thiadiazoles 8a-d was consistent with spectral data (IR, ${ }^{1} \mathrm{H}$ NMR, ${ }^{13} \mathrm{C}$ NMR, and MS (Mass spectroscopy)) and elemental analyses (see Supplementary Materials). As shown in Scheme 2, the reaction proceeded through nucleophilic displacement of the thiol group to the halogen atom to give $S$-alkylated intermediate products $\mathbf{6 a - d}$ [25]. Intramolecular Michael addition [26] of the $\mathrm{NH}$ group into the electrophilic carbon atom of $(\mathrm{C}=\mathrm{N}-\mathrm{N}=)$ for intermediates $\mathbf{6 a}-\mathbf{d}$ led to the formation of cycloadducts $\mathbf{7 a}-\mathbf{d}$. Elimination of ammonia from the latter intermediates $\mathbf{7 a}-\mathbf{d}$ gave the final products 8a-d (Scheme 2).

\section{Conclusions}

Recently, nanoparticles (NPs) have been developed as promising candidates in various applications due to their unique properties. In this article, a chitosan-MgO nanocomposite (as a green recyclable biocatalyst) was prepared and well-characterized using FTIR, FESEM, and EDX spectra. The average size of the $\mathrm{MgO}$ particles was found to be approximately $6-11 \mathrm{~nm}$ for $10 \mathrm{wt} \%$ and it was found that the particles' size slightly decreased with increasing magnesia content. This nanocomposite was then used successfully as a heterogeneous basic catalyst for the synthesis of two series of hydrazonothiazoles and hydrazono $[1,3,4]$ thiadiazoles, with sulfonamide moiety, in a comparative study with triethylamine (as a traditional catalyst). In addition to the preferable green impact, the acquired results showed that the chitosan-MgO nanocomposite was a more powerful catalyst in these reactions as compared to triethylamine. The obvious catalytic potency of the chitosan- $\mathrm{MgO}$ nanocomposite was attributed to the obtained nanosized $\mathrm{MgO}$ and the synergistic effect that is created by the combination of the basic nature of both $\mathrm{MgO}$ and chitosan. Moreover, the nanocatalyst could be easily recovered and reused many times without loss in its catalytic activity. Finally, the biopolymer chitosan-metal oxide combination is a promising hybrid nanocomposite that deserves to be explored in many organic transformations.

Supplementary Materials: The following are available online at http:/ / www.mdpi.com/2079-4991/8/11/928/ s1.

Author Contributions: S.M.R. suggested the plan, interpreted the results, and wrote the chemistry part of the manuscript; K.D.K. prepared the chitosan-MgO nanocomposite, interpreted the results, performed the experiments, and wrote the other part of the manuscript; A.A. revised the manuscript.

Funding: This research received no external funding.

Conflicts of Interest: The authors declare no conflict of interest.

\section{References}

1. Babaie, M.; Sheibani, H. Nanosized magnesium oxide as a highly effective heterogeneous base catalyst for the rapid synthesis of pyranopyrazoles via a tandem four-component reaction. Arab. J. Chem. 2011, 4, 159-162. [CrossRef]

2. Kumar, D.; Reddy, V.B.; Mishra, B.G.; Rana, R.K.; Nadagouda, M.N.; Varma, R.S. Nanosized magnesium oxide as catalyst for the rapid and green synthesis of substituted 2-amino-2-chromenes. Tetrahedron 2007, 63, 3093-3097. [CrossRef] 
3. Mirzaei, H.; Davoodnia, A. Microwave Assisted Sol-Gel Synthesis of MgO Nanoparticles and Their Catalytic Activity in the Synthesis of Hantzsch 1,4-Dihydropyridines. Chin. J. Catal. 2012, 33, 1502-1507. [CrossRef]

4. Al-Matar, H.M.; Khalil, K.D.; Meier, H.; Kolshorn, H.; Elnagdi, M.H. Chitosan as heterogeneous catalyst in Michael additions: The reaction of cinnamonitriles with active methylene Moieties and phenols. Arkivoc 2008, 288-301. [CrossRef]

5. Khalil, K.D.; Al-Matar, H.M.; Elnagdi, M.H. Chitosan as eco-friendly heterogeneous catalyst in Michael type additions: Simple and efficient route to pyridones and phthalazines. Eur. J Chem. 2010, 1, 252-258. [CrossRef]

6. Khalil, K.D.; Al-Matar, H.M. Chitosan based heterogeneous catalyses: 4-Vinylpyridine grafted chitosan as catalyses for Michael additions and alkylpyridazinyl carbonitrile oxidation. Molecules 2013, 18, 5288-5305. [CrossRef] [PubMed]

7. Pramod, K.S.; Praveen, K.S.; Sushil, K.G.; Dau, D.A. Chitosan: An efficient, reusable, and biodegradable catalyst for green synthesis of heterocycles. Ind. Eng. Chem. Res. 2014, 53, 2085-2091. [CrossRef]

8. Sanuja, S.; Agalya, A.; Umapathy, M.J. Studies on Magnesium Oxide Reinforced Chitosan Bionanocomposite Incorporated with Clove Oil for Active Food Packaging Application. Int. J. Polym. Mater. Biopolym. Mater. 2014, 63, 733-740. [CrossRef]

9. Basumallick, S.; Santra, S. Chitosan coated copper-oxide nano particles: A novel electro-catalyst for $\mathrm{CO}_{2}$ reduction. RSC Adv. 2014, 4, 63685-63690. [CrossRef]

10. Guibal, E. Heterogeneous catalysis on chitosan-based materials: A review. Prog. Polym. Sci. 2005, 30, 71-109. [CrossRef]

11. Zablotskaya, A.; Segal, I.; Geronikaki, A.; Eremkina, T.; Belyakov, S.; Petrova, M.; Shestakova, I.; Zvejniece, V.; Nikolajeva, V. Synthesis, physicochemical characterization, cytotoxicity, antimicrobial, anti-inflammatory and psychotropic activity of new $N$-[1,3-(benzo)thiazol-2-yl]- $\omega$-[3,4-dihydroisoquinolin-2(1H)-yl]alkanamides. Eur. J. Med. Chem. 2013, 70, 846-856. [CrossRef] [PubMed]

12. Djukicm, M.; Fesatidou, M.; Xenikakis, I.; Geronikaki, A.; Angelova, V.T.; Savic, V.; Pasic, M.; Krilovic, B.; Djukic, D.; Gobeljic, B.; et al. In vitro antioxidant activity of thiazolidinone derivatives of 1,3-thiazole and 1,3,4-thiadiazole. Chem. Biol. Interact. 2018, 286, 119-131. [CrossRef] [PubMed]

13. Hu, Y.; Li, C.-Y.; Wang, X.-M.; Yang, Y.-H.; Zhu, H.-L. [1,3,4]Thiadiazole: Synthesis, reactions, and applications in medicinal, agriculture, and materials chemistry. Chem. Rev. 2014, 114, 5572-5610. [CrossRef] [PubMed]

14. Riyadh, S.M.; El-Motairi, S.A.; Ahmed, H.E.A.; Khalil, K.D.; Habib, E.E. Synthesis, Biological Evaluation, and Molecular Docking of Novel Thiazoles and [1,3,4]Thiadiazoles Incorporating Sulfonamide Group as DHFR Inhibitors. Chem. Biodiver. 2018, 15, e1800231. [CrossRef] [PubMed]

15. Abbas, E.M.H.; Gomha, S.M.; Farghaly, T.A.; Abdalla, M.M. Synthesis of New Thiazole Derivatives as Antitumor Agents. Curr. Org. Synth. 2016, 13, 456-465. [CrossRef]

16. Al-Bogami, A.S.; Saleh, T.S.; Mekky, A.E.M.; Shaaban, M.R. Microwave assisted regioselective synthesis and 2D-NMR studies of novel azoles and azoloazines utilizing fluorine-containing building Blocks. J. Mol. Struct. 2016, 1121, 167-179. [CrossRef]

17. Eweiss, N.F.; Osman, A. Synthesis of Heterocycles Part II. New Routes to Acetylthiadiazolines and Alkylazothiazoles. J. Heterocycl. Chem. 1980, 17, 1713-1717. [CrossRef]

18. Mosselhi, M.A.N.; Abdallah, M.A.; Mohamed, Y.F.; Shawali, A.S. Synthesis and Tautomeric Structure of 7-Arylhydrazono-7H-[1,2,4]Triazolo[3,4-b][1,3,4]Thiadiazines. Phosphorous Sulfur Silicon 2002, 177, 487-496. [CrossRef]

19. Laude, B.; Soufiaoui, M.; Arriau, J. Cycloadditions dipolaires-1,3 II. Addition des diarylnitrilimines au $\mathrm{N}$-methylindole. Etude experimentale et essai d'interpretation. J. Heterocycl. Chem. 1977, 14, 1183-1190. [CrossRef]

20. Wolkoff, P. A New Method of Preparing Hydrazonyl Halides. Can. J. Chem. 1975, 53, 1333-1335. [CrossRef]

21. El Kadib, A.; Primo, A.; Molvinger, K.; Boumina, M.; Brunel, D. Nanosized vanadium, tungsten, and molybdenium oxide clusters grown in porous chitosan microspheres as promising hybrid materials for selective alcohol oxidation. Chem. A Eur. J. 2011, 17, 7940-7946. [CrossRef] [PubMed]

22. Agrawal, N.R.; Bahekar, S.P.; Agrawal, A.R.; Sarode, P.B.; Chandak, H.S. Cascade Michael-Aldol reaction: Efficient annulation of sulfonamide chalcones into novel cyclohexenones under solvent-free conditions. Arkivoc 2016, 227-245. [CrossRef] 
23. De Oliveira Cardoso, M.V.; de Siqueira, L.R.P.; de Silva, E.B.; Costa, L.B.; Hernandes, M.Z.; Rabello, M.M.; Ferreira, R.S.; de Cruz, L.F.; Moreira, D.R.M.; Pereira, V.R.A.; et al. 2-Pyridyl thiazoles as novel anti-Trypanosoma cruzi agents: Structural design, synthesis and pharmacological evaluation. Eur. J. Med. Chem. 2014, 86, 48-59. [CrossRef] [PubMed]

24. Rostom, S.A.F.; Faidallah, H.M.; Radwan, M.F.; Badr, M.H. Bifunctional ethyl 2-amino-4-methylthiazole5-carboxylate derivatives: Synthesis and in vitro biological evaluation as antimicrobial and anticancer agents. Eur. J. Med. Chem. 2014, 76, 170-181. [CrossRef] [PubMed]

25. Geies, A.A.; Kamal-Eldeen, A.M.; Abdelhafez, A.A.; Gaber, A.M. Synthesis of some thiazolo[3,2-a]pyrimidines. Phosphorous Sulfur Silicon Relat. Elem. 1991, 56, 87-93. [CrossRef]

26. Ranu, B.C.; Banerjee, S. Ionic Liquid as Catalyst and Reaction Medium. The Dramatic Influence of a Task-Specific Ionic Liquid, [bmIm] OH, in Michael Addition of Active Methylene Compounds to Conjugated Ketones, Carboxylic Esters, and Nitriles. Org. Lett. 2005, 7, 3049-3052. [CrossRef] [PubMed]

C 2018 by the authors. Licensee MDPI, Basel, Switzerland. This article is an open access article distributed under the terms and conditions of the Creative Commons Attribution (CC BY) license (http://creativecommons.org/licenses/by/4.0/). 